\title{
Topological and geometric hyperbolicity criteria for polynomial automorphisms of $\mathbb{C}^{2}$
}

\author{
ERIC BEDFORD $\dagger$ and ROMAIN DUJARDIN $\ddagger$ \\ $\dagger$ Institute for Mathematical Sciences, Stony Brook University, \\ Stony Brook, NY 11794, USA \\ (e-mail: ebedford@math.stonybrook.edu) \\ † Sorbonne Université, CNRS, Laboratoire de Probabilités, Statistiques et Modélisations \\ (LPSM), F-75005 Paris, France \\ (e-mail: romain.dujardin@sorbonne-universite.fr)
}

(Received 2 July 2020 and accepted in revised form 23 March 2021)

Abstract. We prove that uniform hyperbolicity is invariant under topological conjugacy for dissipative polynomial automorphisms of $\mathbb{C}^{2}$. Along the way we also show that a sufficient condition for hyperbolicity is that local stable and unstable manifolds of saddle points have uniform geometry.

Key words: polynomial automorphisms, complex Hénon map, hyperbolicity, non-uniform hyperbolicity.

2020 Mathematics Subject Classification: 37F80, 37F15, 37F10 (Primary)

\section{Introduction}

1.1. The main motivation of this paper is to study the following problem.

Question 1.1. Is uniform hyperbolicity a topological property for complex Hénon maps?

We use the terminology 'complex Hénon map' as a synonym for 'polynomial automorphism of $\mathbb{C}^{2}$ with non-trivial dynamics'. Here by non-trivial dynamics we mean that the non-wandering set is not algebraic, or equivalently that the topological entropy is positive. By [FM] we can normalize such a map such that it is a product of actual Hénon maps $(z, w) \mapsto(a w+p(z), a z)$. Hyperbolicity here is understood in the sense of [BS1], that is, we say that a complex Hénon map $f$ is hyperbolic if its Julia set $J=J_{f}$ is a hyperbolic set, which must then be of saddle type. This was shown in [BS1] to have strong consequences on the global dynamics of $f$ : the chain recurrent set consists of $J$ together with finitely many periodic attractors, and $f$ satisfies Smale's Axiom A on $\mathbb{C}^{2}$. (See [I2] for a recent survey on complex Hénon dynamics, with an emphasis on hyperbolic maps.) 
Let us recall a bit of standard notation. Given a complex Hénon map $f$, we denote by $\mathrm{J}^{+}$the forward Julia set, which is the locus of non-normality of forward iterates $\left(f^{n}\right)_{n \geq 0}$, or equivalently the boundary of the set $K^{+}$of points with bounded forward orbits. We likewise define $J^{-}$and $K^{-}$for backward dynamics, and we set $J=J^{+} \cap J^{-}$. We denote by $J^{\star}$ the closure of the set of saddle periodic orbits, which is contained in $J$. For hyperbolic maps, by [BS1] we have $J=J^{\star}$; however, this equality is an open problem in the general case.

Question 1.1 was previously considered for rational maps on the Riemann sphere and for smooth Anosov diffeomorphisms of (real) compact manifolds. Let us start by briefly reviewing these cases.

1.2. One-dimensional rational maps. For polynomials and rational maps in one variable, the answer to the question is 'yes' since there is a simple topological (necessary and sufficient) criterion for hyperbolicity: $\overline{\mathrm{PC}(f)} \cap J=\emptyset$, where $\mathrm{PC}(f)$ is the postcritical set. As a consequence, if $f_{0}$ and $f_{1}$ are rational maps in one variable such that $f_{0}$ is hyperbolic, and $\phi: \mathbb{P}^{1} \rightarrow \mathbb{P}^{1}$ is a topological conjugacy between $f_{0}$ and $f_{1}$, then $f_{1}$ is hyperbolic. Actually the statement already holds locally near the Julia set.

PROPOSITION 1.2. Let $f_{0}$ and $f_{1}$ be rational maps on $\mathbb{P}^{1}$, and assume that $f_{0}$ is hyperbolic. If there are neighborhoods $N\left(J_{0}\right)$ and $N\left(J_{1}\right)$ of $J_{0}$ and $J_{1}$ and a homeomorphism $\phi: N\left(J_{0}\right) \rightarrow N\left(J_{1}\right)$ which satisfies $\phi \circ f_{0}=f_{1} \circ \phi$ wherever these compositions make sense, then $f_{1}$ is hyperbolic.

The result is not obvious since the conjugacy $\phi$ cannot detect that a point in $N\left(J_{1}\right)$ is postcritical. Here and throughout the paper we use indices 0 and 1 to label the dynamical objects (Julia set, etc.) respectively associated to $f_{0}$ and $f_{1}$.

Proof. Notice first that the conjugacy $\phi$ sends periodic points to periodic points. The topological dynamics around a periodic point determines its type (attracting, repelling, neutral) so it follows that all periodic points of $f_{1}$ are hyperbolic. In particular, $f_{1}$ has no parabolic points.

Since the Julia set is the accumulation set of periodic orbits we infer that $\phi\left(J_{0}\right)=J_{1}$. Now a rational map without parabolic points is hyperbolic if and only if its critical set is disjoint from the Julia set. This property holds for $f_{1}$ by topological conjugacy, so the result follows.

Remark 1.3. (1) It is not enough in the proposition to assume that $\phi$ is a conjugating homeomorphism $J_{0} \rightarrow J_{1}$. Indeed, it is well known that $z^{2}+\frac{1}{4}$ is topologically conjugate on its Julia set to any quadratic polynomial in the main cardioid (e.g. $z^{2}$ ). As we will comment below, a similar phenomenon holds for complex Hénon maps (see [RT]).

(2) If we suppose a priori that $\operatorname{deg}\left(f_{0}\right)=\operatorname{deg}\left(f_{1}\right)=d$, we can relax the assumption on $\phi$ by assuming only that $\phi$ is any injective continuous map defined in a neighborhood $N_{0}$ of $J_{0}$ and such that $\phi \circ f_{0}=f_{1} \circ \phi$ wherever these compositions makes sense. Indeed, by the invariance of domain theorem, $\phi\left(N_{0}\right)$ is an open subset of the plane. We only have to show 
that it contains $J\left(f_{1}\right)$. Indeed, $f_{0}$ has only finitely many non-repelling periodic points, so $J_{0}$ contains $p_{n} \sim d^{n}$ repelling periodic points of period $d$ for large $n$. Thus, by topological conjugacy, $\phi\left(J_{0}\right)$ contains $p_{n}$ repelling periodic points of $f_{1}$, which are equidistributed to the equilibrium measure $\mu_{f_{1}}$ whose support is $J\left(f_{1}\right)$. Therefore $\phi\left(J_{0}\right)=J_{1}$ and we are done.

1.3. Anosov diffeomorphisms. The problem of topological invariance of hyperbolicity in real dynamics has been popularized in particular by A. Katok. The answer is already quite subtle for the simplest case of Anosov diffeomorphisms of the 2-torus.

Indeed, there exist examples of $C^{2}$ diffeomorphisms $f$ of the 2-torus which are not hyperbolic but still globally topologically conjugate to a linear Anosov map. This can be done either by carefully deforming a linear Anosov map until some saddle fixed point becomes neutral by preserving the geometry of the stable and unstable foliations (see $[\mathrm{K}]$ ), or by deforming the foliations until reaching a cubic heteroclinic tangency (see [E, BDV]).

If we now require the conjugacy to be Hölder continuous then there are different regimes depending on the precise Hölder regularity. First, it can be arranged that in the previous examples the conjugacy and its inverse are Hölder continuous [Go], and thus hyperbolicity is not invariant under Hölder conjugacy. On the other hand, if the conjugacy is sufficiently close to being bi-Lipschitz-namely, the product of the Hölder exponents of $\phi$ and $\phi^{-1}$ is greater than $1 / 2$ - then $f$ is Anosov [F] (see also [Go]).

1.4. A conjecture. The most natural way to address Question 1.1 would be to find a topological criterion ensuring hyperbolicity for a complex Hénon map, in the spirit of the one-dimensional condition $J \cap \overline{\mathrm{PC}(f)}=\emptyset$. Strictly speaking, a Hénon map admits no critical points; nevertheless there are ways to give a reasonable meaning to this condition-which are more differential-geometric than topological, though. For instance, in the dissipative regime, the condition that there are no critical points on $J$ naturally corresponds to the existence of a dominated splitting and, provided dissipation is strong enough, a good analogue of the one-dimensional situation was achieved in [LP].

A variant is to translate the condition $J \cap \overline{\mathrm{PC}(f)}=\emptyset$ into a regularity property of the geometry of the forward and backward Julia sets $J^{+/-}$near $J$. In this respect it was shown in [BS8] that if in some neighborhood of $J, J^{+}$and $J^{-}$are the supports of two Riemann surface laminations which are transverse along $J$, then $f$ is hyperbolic. In $\S 2$ below we re-prove and generalize this result in several ways.

Let us also point out that several sufficient conditions for hyperbolicity were obtained in [HOV, I1, IS]. In these works, the mechanism for hyperbolicity relies on the complex-analytic geometry of the bidisk, and it is unclear to us whether these conditions should be considered as topological.

Back to our initial problem, even if it is unclear how to design a purely topological criterion for hyperbolicity, one may ask whether hyperbolicity is invariant under topological conjugacy. Here is a precise analogue of Proposition 1.2 for complex Hénon maps. 
Conjecture 1.4. Let $f_{0}$ and $f_{1}$ be two polynomial automorphisms of $\mathbb{C}^{2}$ with non-trivial dynamics, and assume that $f_{0}$ is hyperbolic. Suppose that there exist respective neighborhoods $N_{0}$ and $N_{1}$ of $J_{0}=J_{0}^{\star}$ and $J_{1}^{\star}$ and a homeomorphism $\phi: N_{0} \rightarrow N_{1}$ such that $\phi \circ f_{0}=f_{1} \circ \phi$ wherever these compositions makes sense. Then $f_{1}$ is hyperbolic.

Here are some comments on this conjecture.

(1) It was shown in [D2] (see also [GP]) that for a complex Hénon map hyperbolicity on $J^{\star}$ implies hyperbolicity on $J$. This explains why we can restrict to a neighborhood of $J_{0}^{\star}$ instead of $J_{0}$, and opens the way to an analysis of hyperbolicity based on periodic points.

(2) If we add the hypothesis that $f_{0}$ and $f_{1}$ have the same dynamical degree, then by using the equidistribution of periodic orbits from [BLS2] and arguing as in Remark 1.3 we can relax the assumption that $\phi\left(N\left(J_{0}^{\star}\right)\right)$ contains $J_{1}^{\star}$.

(3) As observed in Remark 1.3, the conjecture is false if the conjugating homeomorphism $\phi$ is only defined on $J_{0}=J_{0}^{\star}$ (see [RT]).

(4) The conjecture is true if $\phi$ is obtained by deformation in the following sense: it was shown in [BD] that if there is a weakly stable holomorphic family $\left(f_{\lambda}\right)$ connecting $f_{0}$ and $f_{1}$, then $f_{1}$ is hyperbolic. (The original statement in [BD] concerns hyperbolicity on $J^{\star}$, so we further use [D2] to deduce hyperbolicity on $J$.)

1.5. Quasi-hyperbolicity. The methods in this paper are closely related to the notion of quasi-hyperbolicity. If $p$ is a saddle point and $r>0$, we let $W_{r}^{s / u}(p)$ denote the connected component of $W^{s / u}(p) \cap B(p, r)$ containing $p$. Following [BS8], a complex Hénon map $f$ is said to be quasi-hyperbolic if there exist positive constants $r$ and $B$ such that for every saddle periodic point $p$ :

(i) $\quad W_{r}^{s / u}(p)$ is closed in $B(p, r)$; and

(ii) the area of $W_{r}^{s / u}(p)$ is bounded by $B$.

If $\phi: N_{0} \rightarrow N_{1}$ is a topological conjugacy as in Conjecture 1.4 then $\phi$ preserves stable and unstable manifolds, so if (i) holds for $f_{0}$ it will also hold for $f_{1}$ (after possibly shrinking $r$ ). It was shown in [BGS] that if $f$ is quasi-hyperbolic then there exist stable and unstable manifolds $\mathcal{W}^{s / u}(x)$ through each point $x \in J^{\star}$. Furthermore, $f$ is uniformly hyperbolic (on $J^{\star}$ and thus $J$ ) if and only if there is no tangency between $\mathcal{W}^{s}$ and $\mathcal{W}^{u}$. Thus if we already know that $f$ is quasi-hyperbolic, then the additional condition of hyperbolicity is a topological invariant in the sense of the conjecture. At this stage, however, it remains an open question whether quasi-hyperbolicity is a topological property.

1.6. Results and outline. In $\S 2$ we establish several sufficient conditions for hyperbolicity based on the geometry of local stable and unstable manifolds of saddle periodic points. A first sufficient condition for hyperbolicity, which essentially follows from [BS8], is that these local stable and unstable manifolds have uniform size and the angle between them is uniformly bounded from below. We give a self-contained proof of this result (see Theorem 2.8). We further show that the transversality assumption is superfluous (Theorems 2.12 and 2.13), and that, as might be expected, in the dissipative case it is enough to control the geometry of unstable manifolds (Theorem 2.18). 
In $\S 3$ we prove Conjecture 1.4 in the case where $f_{1}$ is dissipative (Theorem 3.6). In the conservative case the conjecture holds provided $\phi$ is Hölder continuous (Theorem 3.8). The general case remains open. (Notice that the Jacobian is not invariant under topological conjugacy: the Hénon map $(z, w) \mapsto\left(z^{2}+c+a w, z\right)$ is conjugate to a horseshoe for any Jacobian $a$, as soon as $|c| \gg|a|$.)

\section{Geometric criteria for hyperbolicity}

2.1. Size of a submanifold at a point and $u / s$-regularity. Endow $\mathbb{C}^{2}$ with the Euclidean metric. A bidisk of size $r$ is the image of $D(0, r)^{2}$ under some affine isometry. A curve $V$ in $\mathbb{C}^{2}$ is a graph over an affine line $L$ if the orthogonal projection onto $L$ is injective when restricted to $V$. Then there is a well-defined notion of slope of a holomorphic curve with respect to $L$.

Definition 2.1. A curve $V$ through $p$ is said to have size $r$ at $p$ if there exists a neighborhood of $p$ in $V$ that is a graph of slope at most 1 over a disk of radius $r$ in the tangent space $T_{p} V$.

If $\Delta$ is a disk of size $r$ at $p$, fixing orthonormal coordinates $(x, y)$ so that $p=0$ and $T_{p} V=\{y=0\}$, we get that the connected component of $\Delta$ through $p$ in the bidisk $D(0, r)^{2}$ is a graph $\{y=\varphi(x)\}$ over the first coordinate with $\left|\varphi^{\prime}\right| \leq 1$ and $\varphi^{\prime}(0)=0$. In particular, if $\Delta$ is immersed and has size $r$ at $p$, then it is a submanifold in $B(0, r / \sqrt{2})$ (because a bidisk of size $r$ contains a ball of radius $r / \sqrt{2}$ ).

We now recall a few concepts from [BD]. A point $x \in J^{\star}$ is said $u$-regular (respectively, $s$-regular) if there exist $r>0$ and a sequence of saddle points $\left(p_{n}\right)$ converging to $x$ such that $W^{u}\left(p_{n}\right)$ (respectively, $\left.W^{s}\left(p_{n}\right)\right)$ is of size $r$ at $p_{n}$. In this case it can be shown that the sequence of disks $W_{r}^{u}\left(p_{n}\right)$ (respectively, $\left.W_{r}^{s}\left(p_{n}\right)\right)$ converges in the $C^{1}$ topology to a (smooth) holomorphic disk of size $r$ at $x$ which we denote by $\mathcal{W}_{r}^{u}(x)$ (respectively, $\mathcal{W}_{r}^{s}(x)$ ) (see [BD, Proposition 4.2]). This notation is meant to emphasize that at this stage $\mathcal{W}_{r}^{s / u}(x)$ need not be a stable/unstable manifold in the usual sense. We use the notation $\mathcal{W}_{\text {loc }}^{u / s}(x)$ for an unspecified neighborhood of $x$ in $\mathcal{W}_{r}^{u}(x)$. We say that $x$ is regular if it is $u$ - and $s$-regular and $\mathcal{W}_{\text {loc }}^{u}(x)$ and $\mathcal{W}_{\text {loc }}^{s}(x)$ do not coincide, and transverse regular if they are transverse. In particular, we have the implications

$$
u \text { - and } s \text {-regular } \Leftarrow \text { regular } \Leftarrow \text { transverse regular. }
$$

It is easy to see that if $x$ is a saddle point, then $x$ is regular (indeed, we can pick $p_{n}=x$ or observe that $x$ generates homoclinic intersections, hence it belongs to a horseshoe) and $\mathcal{W}_{\text {loc }}^{s}(x)$ and $\mathcal{W}_{\text {loc }}^{u}(x)$ coincide with the classical local stable and unstable manifolds of $x$. More generally, define a local stable set

$$
V_{\mathrm{loc}, \varepsilon}^{s}(x)=\left\{y: \operatorname{dist}\left(f^{n}(x), f^{n}(y)\right)<\varepsilon \text { for all } n \geq 0 \text {, and } \lim _{n \rightarrow \infty} \operatorname{dist}\left(f^{n}(x), f^{n}(y)\right)=0\right\} .
$$

LEMmA 2.2. Suppose that there is a complex disk $\Delta$ such that $x \in \Delta \subset V_{\mathrm{loc}, \varepsilon}^{s}(x)$. If $x$ is $s$-regular, then $\Delta$ coincides with $\mathcal{W}_{\mathrm{loc}}^{s}(x)$ in a neighborhood of $x$. The analogous result holds with s replaced by $u$. 
Proof. If $\left(p_{n}\right)$ is a sequence of saddle points converging to $x$, then for some $r>0$, $\mathcal{W}_{r}^{s}\left(p_{n}\right)=W_{r}^{S}\left(p_{n}\right)$ must coincide with or be disjoint from $V_{\text {loc, } \varepsilon}^{s}(x)$, and converge to $\mathcal{W}_{r}^{s}(x)$ in the $C^{1}$ topology. Since both $\Delta$ and $\mathcal{W}_{r}^{s}(x)$ contain $x$, by the Hurwitz theorem we conclude that $\Delta$ locally coincides with $\mathcal{W}_{r}^{s}(x)$ and the result follows.

Lemma 2.2 applies in particular to Pesin stable manifolds, so we get the following result (see also [BD, Proposition 4.4]).

LEMMA 2.3. If $x$ is regular in the sense of Pesin theory, and $x$ is $u$-and s-regular in the above sense, then $\mathcal{W}_{\mathrm{loc}}^{s / u}(x)$ agree locally at $x$ with the Pesin manifolds $W_{\mathrm{Pesin}}^{s / u}(x)$. In particular, $x$ is transverse regular.

We say that $x \in J^{\star}$ is uniformly $u$-regular if there exists $r>0$ such that for any sequence $\left(p_{n}\right)$ converging to $x, W^{u}\left(p_{n}\right)$ has size $r$ at $p_{n}$ for sufficiently large $n$. Equivalently, there exists a neighborhood $V$ of $x$ such that for every saddle point $p \in$ $V, W^{u}(p)$ has size $r$ at $p$. If required we can specify the size $r$ in the terminology. Uniform $s$-regularity is defined similarly. Finally, we say that $x$ is uniformly (respectively, transverse) regular if it is uniformly $u$ - and $s$-regular, and regular (respectively, transverse regular).

The following result will play an important role in this paper (of course it admits an identical $s$-regular version).

Proposition 2.4. The following assertions are equivalent.

(1) Every point in $J^{\star}$ is uniformly u-regular.

(2) There exists a uniform $r>0$ such that for every saddle periodic point $p, W^{u}(p)$ has size $r$ at $p$.

(3) There exist a uniform $r>0$ and a dense set $D$ of saddle periodic points such that for every $p \in D, W^{u}(p)$ has size $r$ at $p$.

(4) There exists a lamination $\mathcal{W}^{u}$ by Riemann surfaces in a neighborhood of $J^{\star}$ which extends the family of local unstable manifolds of saddle points.

Proof. (1) implies (2) by a simple compactness argument. The implications (2) $\Rightarrow$ (3) and $(4) \Rightarrow(1)$ are obvious, and $(3) \Rightarrow$ (4) follows from standard holomorphic motion techniques. Let us give some details on this last point for the reader's convenience (see also [BD, Proposition 4.2 and Lemma 5.3]). We start with the following basic geometric idea. If $\Delta$ and $\Delta^{\prime}$ are flat disks in $B(0, r / 10) \subset \mathbb{C}^{2}$ which both intersect $B(0, r / 1000)$, and whose tangent vectors are 1/4-far apart (relative to the usual Fubini-Study metric on $\mathbb{P}^{1}$ ), then $\Delta$ and $\Delta^{\prime}$ intersect and

$$
\operatorname{dist}\left(\Delta \cap \partial B(0, r / 10), \Delta^{\prime} \cap \partial B(0, r / 10)\right)>\frac{1}{100} .
$$

By the persistence of proper intersections, the same holds for $\widetilde{\Delta}$ and $\widetilde{\Delta}^{\prime}$, whenever $\widetilde{\Delta}$ and $\widetilde{\Delta}^{\prime}$ are holomorphic disks which are respectively $1 / 100$-close to $\Delta$ and $\Delta^{\prime}$. Now if $\Delta$ is a disk of size $r$ at $x$, by the Schwarz lemma, $\Delta \cap B(0, r / 10)$ remains $1 / 100$-close to $T_{x} \Delta$. Taking the contrapositive, we see that if $\Delta$ and $\Delta^{\prime}$ are disks of size $r$ respectively at $x$ and 
$x^{\prime}$, with $\operatorname{dist}\left(x, x^{\prime}\right)<1 / 1000$, then their tangent spaces must be $1 / 4$-close to each other; in particular, they are graphs over a disk of radius $r / 4$ relative to the same orthogonal projection.

Now by (3), for every $x \in J^{\star}$ there is a holomorphic disk $\mathcal{W}_{r}^{u}(x)$ of size $r$ through $x$ and these disks are either disjoint or locally coincide because local unstable manifolds of saddle points are disjoint. By the previous discussion, the disks $\mathcal{W}_{r / 4}^{u}(y)$ are disjoint graphs over some direction for $y$ close to $x$, so they form a lamination by the lambda lemma of [MSS]. Thus we get the desired lamination structure in the $r / 5$-neighborhood of $J^{\star}$.

Remark 2.5. Under the assumptions of Proposition 2.4, there exist a neighborhood $\mathcal{N}$ of $J^{\star}$ and a lamination $\mathcal{W}^{u}$ of $\mathcal{N}$ by Riemann surfaces which extends the family of local unstable manifolds of saddle points. Beware, however, that this does not a priori imply that $J^{-} \cap \mathcal{N}$ is laminated or that it coincides with $\operatorname{Supp}\left(\mathcal{W}^{u}\right)$ : indeed, $J^{-}$is the closure of global unstable manifolds, which could recur to $\mathcal{N}$ in a complicated fashion (this point is an important issue in [D2]).

2.2. Existence of invariant laminations and hyperbolicity. Recall that a complex Hénon map $f$ is said to be hyperbolic if $J$ is a hyperbolic set. As was noted above, by [D2] (see also [GP]) it is actually enough to check hyperbolicity on $J^{\star}$ : this opens the way to hyperbolicity criteria based on periodic points.

THEOREM 2.6. [D2] If $J^{\star}$ is a hyperbolic set for $f$, then $f$ is hyperbolic.

A geometric criterion for hyperbolicity based on the existence and transversality of unstable laminations was established in [BS8, Theorem 8.3]. By incorporating the result of Theorem 2.6 it reads as follows.

THEOREM 2.7. [BS8] Let $f$ be a complex Hénon map. Assume that there exist a neighborhood of $J^{\star}$ and Riemann surface laminations $\mathcal{L}^{ \pm}$of $J^{ \pm}$such that $\mathcal{L}^{+}$and $\mathcal{L}^{-}$ intersect transversally at all points of $J^{\star}$. Then $f$ is hyperbolic.

It is convenient to formulate this result in the language of uniform regularity. The following is an essentially equivalent statement (see, however, Remark 2.5).

THEOREM 2.8. Let $f$ be a complex Hénon map. If every point in $J^{\star}$ is uniformly regular and transverse then $f$ is hyperbolic.

Let us give a self-contained proof of this theorem, which basically follows the approach of [BS8, Theorem 8.3]. First, recall from Proposition 2.4 that if every point in $J^{\star}$ is uniformly $u$-regular, then there exist $r>0$ and a lamination $\mathcal{W}^{u}$ in the $r$-neighborhood of $J^{\star}$, extending the unstable manifolds of saddle points. Recall also the dynamical Green function $G^{+}$, defined by $G^{+}(x)=\lim _{n \rightarrow \infty} d^{-n} \log ^{+}\left\|f^{n}(x)\right\|$. It is a non-negative continuous plurisubharmonic function in $\mathbb{C}^{2}$, with the property that $\left\{G^{+}=0\right\}=K^{+}$. 
Proposition 2.9. Let $f$ be a complex Hénon map. Assume that every point in $J^{\star}$ is uniformly u-regular and that for every $x \in J^{\star},\left.G^{+}\right|_{\mathcal{W}} ^{u}(x) \not \equiv 0$. Then $f$ is uniformly expanding in the direction of $T \mathcal{W}^{u}$ along $J^{\star}$.

This condition on $G^{+}$will be used several times in the sequel; let us emphasize that it means that $G^{+}$does not vanish identically on any neighborhood of $x$ in $\mathcal{W}^{u}(x)$ (see also the notion of $u$-exposedness in [BD, Definition 4.5]).

Proof. Let $r$ be such that for any saddle point $p, W^{u}(p)$ has size $5 r$ at $p$. Then, by Proposition 2.4, $\mathcal{W}^{u}$ defines a lamination in the $r$-neighborhood of $J^{\star}$ such that for every saddle point $p, \mathcal{W}^{u}(p)$ coincides with the local unstable manifold of $p$.

We have to show that $f$ is uniformly expanding along $\left.\mathcal{W}^{u}\right|_{J^{\star}}$, that is, there exist $C>0$ and $\lambda>1$ such that for every $x \in J^{\star}$, every $k \geq 1$ and $e \in T_{x} \mathcal{W}^{u}(x),\left|D f_{x}^{k}(e)\right| \geq C \lambda^{k}|e|$ (where $|\cdot|$ denotes the Riemannian metric induced by the standard Hermitian structure of $\mathbb{C}^{2}$ ). By continuity it is enough to prove this property on the (dense) set $\mathcal{S}$ of saddle periodic points. For this, we will construct a metric $\mid \cdot l^{\prime}$ on $\left.T \mathcal{W}^{u}\right|_{\mathcal{S}}$ which is equivalent to the ambient one (with uniform constants) and such that for every $p \in \mathcal{S}$, and $e \in T_{p} \mathcal{W}^{u}(p)$, $\left|D f_{p}(e)\right|^{\prime} \geq \lambda|e|^{\prime}$.

For every saddle point $p$, the global unstable manifold is biholomorphic to $\mathbb{C}$, so its uniformization $\psi_{p}^{u}: \mathbb{C} \rightarrow W^{u}(p)$ is unique up to a multiplicative factor at the source. In particular, $f$ is affine in these parameterizations, and there is a well-defined notion of a round disk in $W^{u}(p)$, which is $f$-invariant. For $e \in T_{p} W^{u}(p)$ and $\eta>0$ we define $|e|_{\eta}$ in the style of the Kobayashi metric:

$$
|e|_{\eta}=\frac{1}{\rho_{\eta}}
$$

where $\rho_{\eta}=\sup \left\{\rho, \sup _{D(0, \rho)} G^{+} \circ \psi_{p}^{u} \leq \eta\right.$ where $\psi_{p}^{u}: \mathbb{C} \stackrel{\sim}{\rightarrow} W^{u}(p)$ and $\left.\left(\psi_{p}^{u}\right)^{\prime}(0)=e\right\}$.

For every $x \in J^{\star}$ (not necessarily a saddle), $G^{+} \mid \mathcal{W}_{\text {loc }}^{u}(x)$ is not identically 0 near $x$ so we infer that for every $r>0$, sup $G^{+} \mid \mathcal{W}_{r}^{u}(x)>0$. From the continuity of the Green function $G^{+}$, the compactness of $J^{\star}$, and the lamination structure we infer the existence of constants $r>0$ and $\eta_{1}^{\prime}>\eta_{1}>0$ such that for every $x \in J^{\star}$,

$$
\eta_{1} \leq \sup \left(G^{+} \mid \mathcal{W}_{r}^{u}(x)\right) \leq \eta_{1}^{\prime}
$$

Recall that for near any $x \in J^{\star}$, up to a unitary change of coordinates, $\mathcal{W}^{u}$ is a union of graphs over a disk of size $r$ and slope bounded by 1 (relative to some projection $\pi$ ). Thus if $p \in \mathcal{S}$ is close to $x$, and $\psi_{p}^{u}$ is as above, it follows that $\left.\pi \circ \psi_{p}^{u}\right|_{D\left(0, \rho_{\eta_{1}}\right)}$ is a univalent holomorphic function. Set $\eta_{2}=\eta_{1} / 2$. The Koebe distortion theorem and the uniform continuity of the Green function together imply that for $\rho<\rho_{\eta_{2}}, \pi \circ \psi_{p}^{u}(D(0, \rho))$ is approximately a round disk, with uniform distortion bounds. From this uniformity, we infer that there exists $\lambda>1$ such that for every $p \in \mathcal{S}$ and $e \in T_{p} \mathcal{W}^{u}(p),|e|_{\eta_{2} / d} \geq \lambda|e|_{\eta_{2}}$. The invariance relation of the Green function $G^{+}$implies that $\left|d f_{p}(e)\right|_{\eta_{2}}=|e|_{\eta_{2} / d}$. Putting these two facts together, we get that for every $p \in \mathcal{S}$ and $e \in T_{p} \mathcal{W}^{u}(p),\left|d f_{p}(e)\right|_{\eta_{2}} \geq$ 
$\lambda|e|_{\eta_{2}}$. Finally, again from the uniform continuity of the Green function and bounded distortion, we get that $|\cdot|_{\eta_{2}}$ is (uniformly) equivalent to $|\cdot|$ on $\left.T \mathcal{W}^{u}\right|_{\mathcal{S}}$, and the proof is complete.

Remark 2.10. By a standard procedure, up to reducing $\lambda$ it is possible to construct a continuous metric $|\cdot|^{\prime \prime}$ on $\left.T \mathcal{W}^{u}\right|_{J^{\star}}$ such that $\left|D f_{x}(\cdot)\right|^{\prime \prime} \geq \lambda|\cdot|^{\prime \prime}$. Indeed, for a small $\varepsilon>0$ and $e \in T_{x} \mathcal{W}^{u}(x)$, put

$$
|e|^{\prime \prime}=\sum_{n=0}^{\infty}(\lambda-\varepsilon)^{n}\left|D f_{x}^{-n}(e)\right| .
$$

Then one easily checks that $|\cdot|^{\prime \prime}$ is well defined, continuous, and satisfies

$$
\left|D f_{x}^{-1}(e)\right|^{\prime \prime} \leq(\lambda-\varepsilon)^{-1}|e|^{\prime \prime} .
$$

The next result implies that if $f$ is uniformly regular, then the second assumption of Proposition 2.9 holds.

Proposition 2.11. (See [BD, Proposition 4.7]) Let $f$ be a complex Hénon map. If $x \in J^{\star}$ is regular then $\left.G^{+}\right|_{\mathcal{W}_{\mathrm{loc}}^{u}(x)} \neq \equiv 0$.

Proof. If $\left(p_{n}\right)$ is a sequence of distinct saddle points converging to $x$, then $W_{r}^{s}\left(p_{n}\right)$ is a sequence of disjoint submanifolds converging to $\mathcal{W}_{r}^{s}(x)$. Since by assumption $\mathcal{W}_{r}^{u}(x)$ and $\mathcal{W}_{r}^{s}(x)$ are distinct, then for large $n, W_{r}^{s}\left(p_{n}\right)$ must possess transverse intersection points with $\mathcal{W}_{r}^{u}(x)$ close to $x$ : if $\mathcal{W}_{r}^{u}(x)$ and $\mathcal{W}_{r}^{s}(x)$ are transverse this is clear, and if they are tangent this follows from [BLS1, Lemma 6.4]. Then the inclination lemma implies that $\left(\left.f^{n}\right|_{\mathcal{W}} ^{u}(x)\right)$ is not a normal family of holomorphic mappings, therefore $G^{+}$is not harmonic on $\mathcal{W}_{\text {loc }}^{u}(x)$, thus not identically zero, and we are done.

Proof of Theorems 2.7 and 2.8. Under the assumptions of Theorem 2.8, it follows directly from Propositions 2.9 and 2.11 that $f$ is uniformly expanding along $\left.T \mathcal{W}^{u}\right|_{J^{\star}}$ and contracting along $\left.T \mathcal{W}^{s}\right|_{J^{\star}}$, so by the transversality assumption, $J^{\star}$ is a hyperbolic set. Then we conclude from Theorem 2.6 that $f$ is hyperbolic.

To establish Theorem 2.7, it is enough to check that the existence of the transverse laminations $\mathcal{L}^{+}$and $\mathcal{L}^{-}$implies uniform transverse regularity. We first observe that for any saddle point $p, W^{s}(p)$ locally coincides with the leaf $\mathcal{L}^{+}(p)$ of $\mathcal{L}^{+}$through $p$, and likewise in the unstable direction. Indeed, since the leaves of $\mathcal{L}^{+}$are contained in $J^{+}$, for every disk $\Delta$ contained in such a leaf, $\left(\left.f^{n}\right|_{\Delta}\right)_{n \geq 0}$ is a normal family. Now if $\mathcal{L}_{\text {loc }}^{+}(p) \neq W_{\text {loc }}^{s}(p)$ then a first possibility is that they are transverse, and it follows from the inclination lemma that $\left(\left.f^{n}\right|_{\mathcal{L}_{\text {loc }}^{+}(p)}\right)_{n \geq 0}$ is not normal. Otherwise by [BLS1, Lemma 6.4] for any $x \in J^{\star}$ close to $p, \mathcal{L}_{\text {loc }}^{+}(x)$ is transverse to $W_{\text {loc }}^{s}(p)$ and similarly $\left(\left.f^{n}\right|_{\mathcal{L}_{\text {loc }}^{+}(x)}\right)_{n \geq 0}$ is not a normal family. In both cases we reach a contradiction. It then follows from Proposition 2.4 that every point in $J^{\star}$ is uniformly regular and transverse and we conclude as before.

It turns out that the transversality assumption in Theorem 2.7 is unnecessary, that is, uniform regularity rules out the possibility of tangencies. 
THEOREM 2.12. Let $f$ be a complex Hénon map. If every point in $J^{\star}$ is uniformly regular then $f$ is uniformly hyperbolic.

Proof. By Proposition 2.4 there exist laminations $\mathcal{W}^{u}$ and $\mathcal{W}^{s}$ in a neighborhood of $J^{\star}$ extending the family of local stable and unstable manifolds of periodic points, and by Proposition 2.9 and 2.11 we get that $f$ is uniformly expanding along $\mathcal{W}^{u}$ and $f^{-1}$ is uniformly expanding along $\mathcal{W}^{s}$. To prove the theorem we thus have to show that these laminations are transverse at all points of $J^{\star}$. Let $\mathcal{T}$ be the tangency locus, that is, the set of points $x \in J^{\star}$ such that $\mathcal{W}^{s}(x)$ and $\mathcal{W}^{u}(x)$ are tangent at $x$. This is a closed invariant set. Assume by way of contradiction that it is non-empty. Then it supports an ergodic invariant measure $v$. Let

$$
\chi^{+}=\lim _{n \rightarrow+\infty} \frac{1}{n} \int \log \left\|d f_{x}^{n}\right\| d v(x) \quad \text { and } \quad \chi^{-}=\lim _{n \rightarrow+\infty} \frac{1}{n} \int \log \left\|d f_{x}^{-n}\right\|^{-1} d \nu(x)
$$

be the Lyapunov exponents of $v$. Since $f$ is uniformly expanding/contracting along $\mathcal{W}^{u / s}$ we infer that $\chi^{-}<0<\chi^{+}$. By the Oseledets theorem, there exists an associated invariant measurable decomposition $T_{x} \mathbb{C}^{2}=E^{-}(x) \oplus E^{+}(x)$ defined $v$-almost everywhere such that the growth rate of vectors in $E^{ \pm}(x)$ is governed by $\chi^{ \pm}$. By Pesin's theory (see, for example, [FHY]) for $v$-almost every (a.e.) $x$ there are local stable and unstable manifolds $W_{\text {Pesin }}^{s}(x)$ and $W_{\text {Pesin }}^{u}(x)$ respectively tangent to the characteristic directions associated to the negative and positive exponent. But by Lemma 2.3, $W_{\mathrm{Pesin}}^{s / u}(x)$ locally coincides with $\mathcal{W}^{s / u}(x)$, so we infer that $E^{+}(x)=E^{-}(x)$ almost everywhere, which contradicts the Oseledets theorem. This contradiction finishes the proof.

If $f$ is not volume preserving we can further relax the previous criterion.

THEOREM 2.13. Let $f$ be a complex Hénon map with $|\operatorname{Jac}(f)| \neq 1$. If every point in $J^{\star}$ is uniformly $u$-and s-regular then $f$ is uniformly hyperbolic.

Proof. The difference with Theorem 2.12 is that $\mathcal{T}$ can now contain local leaves so Proposition 2.11 does not apply. Let $r$ be the uniform size of local $s / u$-manifolds along $J^{\star}$. Without loss of generality assume that $|\operatorname{Jac}(f)|<1$. Note that if $\mathcal{W}_{\text {loc }}^{u}(x)=\mathcal{W}_{\text {loc }}^{s}(x)$ then $\mathcal{W}_{r}^{u}(x)=\mathcal{W}_{r}^{s}(x)$. Denote by $\mathcal{T}^{\prime}$ the set of points $x \in J^{\star}$ such that $\mathcal{W}_{r}^{u}(x)=\mathcal{W}_{r}^{s}(x)$. Then $\mathcal{T}^{\prime}$ is also closed and invariant. Indeed, if $\mathcal{W}_{r}^{u}(x)=\mathcal{W}_{r}^{s}(x)$ then clearly $\mathcal{W}_{\text {loc }}^{u}(f(x))=$ $\mathcal{W}_{\text {loc }}^{s}(f(x))$, so $\mathcal{W}_{r}^{u}(f(x))=\mathcal{W}_{r}^{s}(f(x))$. Thus $f\left(\mathcal{T}^{\prime}\right) \subset \mathcal{T}^{\prime}$, and the closedness of $\mathcal{T}^{\prime}$ follows directly from the continuity of $x \mapsto \mathcal{W}_{r}^{u / s}(x)$.

Assume by way of contradiction that $\mathcal{T}^{\prime}$ is non-empty. Then it supports an ergodic invariant measure $v$. Since $f$ is dissipative its Lyapunov exponents satisfy $\chi^{-}<0 \leq \chi^{+}$. For every $x \in \mathcal{T}^{\prime}, \mathcal{W}_{r}^{u}(x)=\mathcal{W}_{r}^{s}(x)$ is contained in $J^{+} \cap J^{-}$so it is a Fatou disk under forward and backward iteration. The following lemma relates these disks to the Oseledets decomposition.

LEMMA 2.14. Let $f$ be a complex Hénon map and $v$ be an ergodic invariant measure whose Lyapunov exponents satisfy $\chi^{-}<0 \leq \chi^{+}$, and $T_{x} \mathbb{C}^{2}=E^{-}(x) \oplus E^{+}(x)$ be the associated measurable decomposition. If v-a.e. point is u-regular then for v-a.e. $x$, $\mathcal{W}_{\mathrm{loc}}^{u}(x)$ is tangent to $E^{+}(x)$ at $x$. 
Assuming this result for the moment, let us conclude the proof. The contradiction hypothesis implies that for v-a.e. $x, \mathcal{W}_{\text {loc }}^{u}(x)=\mathcal{W}_{\text {loc }}^{s}(x)$. By Pesin's theory a $v$-generic point $x$ admits a local strong stable manifold $W_{\text {Pesin }}^{s}(x)$, which is tangent to $E^{-}(x)$, and by Lemma 2.3 it coincides with $\mathcal{W}_{\text {loc }}^{s}(x)$. On the other hand, by Lemma $2.14, \mathcal{W}_{\text {loc }}^{u}(x)$ is almost surely transverse to $E^{-}(x)$. This contradiction shows that $\mathcal{T}^{\prime}$ is empty. Therefore every point in $J^{\star}$ is regular, and applying Theorem 2.12 finishes the proof.

Proof of Lemma 2.14. Since $\mathcal{W}_{\mathrm{loc}}^{u}(x)$ is contained in $J^{-},\left(\left.f^{-n}\right|_{\mathcal{W}_{\mathrm{loc}}^{u}(x)}\right)$ is a normal family, so it follows from the Cauchy estimates that $\left\|d f_{x}^{-n}\left(e^{u}(x)\right)\right\|$ is bounded, where $e^{u}(x)$ is any tangent vector to $\mathcal{W}^{u}(x)$ at $x$. On the other hand the Oseledets theorem asserts that almost surely, if $e(x)$ is any non-zero vector such that $e(x) \notin E^{+}(x),\left\|d f_{x}^{-n}(e(x))\right\|$ grows exponentially at rate $\left|\chi^{-}\right|$. Hence $e^{u}(x) \in E^{+}(x)$ and we are done.

2.3. Unstable lamination, dominated splitting and hyperbolicity. It is natural to expect that in the dissipative setting, uniform $u$-regularity is enough to characterize hyperbolicity. Indeed, uniform $u$-regularity should provide uniform expansion along some field of directions, which, together with volume contraction, yields uniform hyperbolicity. The basic technical tool needed to implement this idea is that of dominated splitting. Recall that a dominated splitting on some invariant set $\Lambda$ is a splitting of the form $\left.T \mathbb{C}^{2}\right|_{\Lambda}=E^{s} \oplus E^{c}$ for which there exist $C>0$ and $\lambda<1$ such that

$$
\frac{\left\|\left.d f^{n}\right|_{E^{s}}\right\|}{\left\|\left.d f^{n}\right|_{E^{c}}\right\|} \leq C \lambda^{n} .
$$

Then this splitting is automatically continuous, and if $|\operatorname{Jac}(f)| \leq 1$, the direction $E^{s}$ is contracting. The existence of a dominated splitting for $f$ along $J$ is a way to formalize the 'absence of critical points' on $J$.

Our first result can be viewed as a version of [LP] in a (greatly) simplified setting.

PROPOSITION 2.15. Let $f$ be a complex Hénon map with $|\operatorname{Jac}(f)| \leq 1$. If every point in $J^{\star}$ is uniformly u-regular and if $f$ admits a dominated splitting on $J^{\star}$, then $f$ is hyperbolic.

Proof. Dominated splitting implies the existence of a strong stable lamination $\mathcal{W}^{s}$ in a neighborhood of $J^{\star}$, hence points of $J^{\star}$ are uniformly $s$-regular. Then if $|\operatorname{Jac}(f)|<1$, the result follows directly from Theorem 2.13. In the general case we just have to repeat the proof of Theorem 2.13, the only difference being that dissipativity was used there to show that $v$ has a negative exponent while here this follows from the dominated splitting assumption.

The idea of dominated splitting shows that hyperbolicity already holds under the assumptions of Proposition 2.9.

PROPOSITION 2.16. Let $f$ be a complex Hénon map with $|\operatorname{Jac}(f)| \leq 1$. If every point in $J^{\star}$ is uniformly $u$-regular and, for every $x \in J^{\star},\left.G^{+}\right|_{\mathcal{W}}{ }_{\mathrm{loc}}(x) \neq \equiv$, then $f$ is hyperbolic. 
Applying Proposition 2.11 yields the following corollary, which generalizes (and gives a new approach to) Theorem 2.12.

COROllary 2.17. Let $f$ be a complex Hénon map with $|\operatorname{Jac}(f)| \leq 1$. If every point in $J^{\star}$ is regular and uniformly $u$-regular then $f$ is hyperbolic.

Proof of Proposition 2.16. By the cone criterion for dominated splitting (see [S, Proposition 2.2]) if we can show that for every $x \in J^{\star}$ there exists a cone $\mathcal{C}_{x}$ about $T_{x} \mathcal{W}^{u}(x)$ in $T_{x} \mathbb{C}^{2}$ such that the field of cones $\left(\mathcal{C}_{x}\right)_{x \in J^{\star}}$ is strictly contracted by the dynamics, then the result follows from Proposition 2.15. By Proposition 2.9 and Remark 2.10 there is a continuous Riemannian metric on $\left.T \mathcal{W}^{u}\right|_{J^{\star}}$ which is immediately expanded by the dynamics. Let $\left(e_{x}\right)_{x \in J^{\star}}$ be a field of tangent vectors to $\mathcal{W}^{u}$ of unit norm relative to this metric, and $f_{x}$ be orthogonal to $e_{x}$ in $T_{x} \mathbb{C}^{2}$ (relative to the ambient Riemannian structure) and such that $\operatorname{det}\left(e_{x}, f_{x}\right)=1$. For small $\varepsilon$, define a continuous field of cones $\mathcal{C}_{x}^{\varepsilon} \subset T_{x} \mathbb{C}^{2}$ by

$$
\mathcal{C}_{x}^{\varepsilon}=\left\{u e_{x}+v f_{x},|v| \leq \varepsilon|u|\right\} .
$$

Working in the frame $\left\{\left(e_{x}, f_{x}\right), x \in J^{\star}\right\}$, the matrix expression of $d f_{x}$ is of the form

$$
\left(\begin{array}{cc}
\lambda_{x} & a(x) \\
0 & \lambda_{x}^{-1} J
\end{array}\right),
$$

where $\left|\lambda_{x}\right| \geq \lambda_{0}>1$ and $J$ is the Jacobian, so $|J| \leq 1$. Since the frame $\left(e_{x}, f_{x}\right)$ is continuous, $a(\cdot)$ is bounded. Then one checks easily that if $\varepsilon$ is so small that $\lambda_{0}-\varepsilon\|a\|>1$, then

$$
d f_{x}\left(\mathcal{C}_{x}^{\varepsilon}\right) \subset \mathcal{C}_{f(x)}^{\lambda_{0}^{-1} \varepsilon}
$$

Hence the field of cones $\left(C_{x}\right)_{x \in J^{\star}}$ is strictly contracted by the tangent dynamics and we are done. (Note that a similar argument appears in [D1].)

The next result shows that uniform expansion can indeed be deduced from the geometric property of uniform $u$-regularity. Assume that every $x \in J^{\star}$ is uniformly regular of size $4 r$. Recalling the construction of global unstable manifolds from local ones, for $x \in J^{\star}$ we define

$$
\mathcal{W}^{u}(x)=\bigcup_{n \geq 0} f^{n}\left(\mathcal{W}_{r}^{u}\left(f^{-n}(x)\right)\right) .
$$

It follows from this definition that $f^{-1}\left(\mathcal{W}^{u}(x)\right)=f^{-1}\left(\mathcal{W}_{r}^{u}(x)\right) \cup \mathcal{W}^{u}\left(f^{-1}(x)\right)$, hence $f^{-1}\left(\mathcal{W}^{u}(x)\right)$ contains $\mathcal{W}^{u}\left(f^{-1}(x)\right)$ and it is not a priori clear that the $\mathcal{W}^{u}(x)$ define an invariant family of curves. However, if $\mathcal{W}^{u}(x)$ is biholomorphic to $\mathbb{C}$ for every $x \in J^{\star}$ then $f^{-1}\left(\mathcal{W}^{u}(x)\right)=\mathcal{W}^{u}\left(f^{-1}(x)\right)$, for otherwise $\mathcal{W}^{u}\left(f^{-1}(x)\right)$ would strictly contain $f^{-1}\left(\mathcal{W}^{u}(x)\right)$, and it would be a complex submanifold of $\mathbb{C}^{2}$ biholomorphic to the Riemann sphere, which is contradictory. The following theorem confirms the expectation that the parabolicity of leaves in $J^{-}$is associated with expansion (compare, for example, [LM, §4]). 
THEOREM 2.18. Let $f$ be a dissipative complex Hénon map. If every point $x \in J^{\star}$ is uniformly $u$-regular and in addition $\mathcal{W}^{u}(x)$ is biholomorphic to $\mathbb{C}$, then $f$ is hyperbolic.

Remark that the definition of $\mathcal{W}^{u}(x)$ in (1) a priori depends on $r$. The theorem shows that if these manifolds are biholomorphic to $\mathbb{C}$, this is actually not the case.

Proof. For every $x \in J^{\star}$, fix a uniformization $\psi_{x}^{u}: \mathbb{C} \stackrel{\sim}{\rightarrow} \mathcal{W}^{u}(x)$ such that $\psi_{x}^{u}(0)=x$, which is normalized by $\left|\left(\psi^{u}\right)^{\prime}(0)\right|=1$. For $\eta>0$, define $R_{\eta}(x)$ to be the maximal radius of a round disk in $\mathbb{C}$ such that $\left.G^{+} \circ \psi_{x}^{u}\right|_{D\left(0, R_{\eta}(x)\right)} \leq \eta$ (this is similar but not identical to the definition of $\rho_{\eta}$ in Proposition 2.9). Since $\mathcal{W}^{u}(x)$ is an entire curve contained in $J^{-},\left.G^{+}\right|_{\mathcal{W}^{u}(x)}$ is unbounded so $R_{\eta}(x)$ is finite. We claim that for every $\eta>0$, there exists $C_{\eta}>0$ such that

$$
\text { for every } x \in J^{\star}, \quad C_{\eta}^{-1} \leq R_{\eta}(x) \leq C_{\eta} .
$$

Indeed, fix $x \in J^{\star}$ and let us show that $R_{\eta}$ is locally uniformly bounded from above and below in a neighborhood of $x$. Then by compactness these bounds will be uniform on $J^{\star}$. Viewed in the unstable parameterizations $f$ is affine so it maps circles to circles. Let $\Delta^{u}(x, R)=\psi_{x}^{u}(D(0, R))$. We first claim that there exists $k \geq 0$ such that $f^{-k}\left(\Delta^{u}\left(x, R_{\eta}(x)\right)\right.$ is contained in $\mathcal{W}_{r}^{u}\left(f^{-k}(x)\right)$. Indeed, by definition of $\mathcal{W}^{u}(x)$, for every $x^{\prime} \in \partial \Delta^{u}\left(x, 4 R_{\eta}(x)\right)$, there exists $k \geq 0$ such that $f^{-k}\left(x^{\prime}\right) \in \mathcal{W}_{r}^{u}\left(f^{-k}(x)\right)$. As in the proof of Proposition 2.9, the Koebe distortion theorem implies that there is a coordinate $\pi: \mathcal{W}_{4 r}^{u}\left(f^{-k}(x)\right) \rightarrow \mathbb{C}$ such that that for $s \leq r, \pi\left(\Delta^{u}\left(f^{-k}(x), s\right)\right)$ is approximately a disk of radius $s$. Now $f^{-k}\left(\Delta^{u}\left(x, R_{\eta}(x)\right)\right)$ is a round disk in the affine coordinate, and it possesses a boundary point in $\mathcal{W}_{r / 2}^{u}\left(f^{-k}(x)\right)$, so it follows that it is completely contained in $\mathcal{W}_{r}^{u}\left(f^{-k}(x)\right)$. Therefore, replacing $x$ by $f^{-k}(x)$, we can assume that $\psi_{x}^{u}\left(D\left(0, R_{\eta}\right)\right) \subset$ $\mathcal{W}_{r}^{u}(x)$.

By uniform $u$-regularity, for $y$ close to $x, \mathcal{W}_{r}^{u}(y)$ is a graph of slope at most 1 over a disk of size $r$ relative to the projection $\pi$. Thus from Koebe distortion again, we infer that for $y$ close to $x$, the distance induced by the normalized affine structure along the $\mathcal{W}_{r}^{u}(y)$ is equivalent to the ambient distance. In particular, there exists a constant $K$ depending only on $r$ such that for $y$ close to $x$ and $\eta$ as above,

$$
K^{-1} \operatorname{dist}\left(y,\left\{G^{+}=\eta\right\}\right) \leq R_{\eta}(y) \leq K \operatorname{dist}\left(y,\left\{G^{+}=\eta\right\}\right) .
$$

Finally, by the Hölder continuity of $G^{+}, \operatorname{dist}\left(y,\left\{G^{+}=\eta\right\}\right)$ is bounded from below by $C \eta^{\theta}$, and if $\operatorname{dist}(y, x) \leq r$ it is bounded from above by $C r$. This completes the proof of (2).

Then, from the invariance relation of $G^{+}$, we have

$$
f\left(\Delta^{u}\left(x, R_{\eta}(x)\right)\right)=\Delta^{u}\left(f(x), R_{d \eta}(f(x))\right) \supset \Delta^{u}\left(f(x), R_{\eta}(f(x))\right),
$$

hence for every $n \geq 0$ we infer that $f^{n}\left(\Delta^{u}\left(x, R_{\eta}(x)\right)\right) \supset \Delta^{u}\left(f^{n}(x), R_{\eta}\left(f^{n}(x)\right)\right)$. In particular, for every $x \in J^{\star}$ and every $n \geq 1$ we have that that $f^{n}\left(\Delta^{u}\left(x, C_{\eta}\right)\right) \supset$ $\Delta^{u}\left(f^{n}(x), C_{\eta}^{-1}\right)$. Again since $f$ is affine in the unstable parameterizations we deduce that for every $t>0$,

$$
f^{n}\left(\Delta^{u}\left(x, t C_{\eta}\right)\right) \supset \Delta^{u}\left(f^{n}(x), t C_{\eta}^{-1}\right) .
$$

Finally, taking the derivative at $t=0$, we conclude that $\left\|\left.D f_{x}^{n}\right|_{T_{x}} \mathcal{W}^{u}\right\| \geq\left(C_{\eta}\right)^{-2}$. 
This bound in turns implies the existence of a dominated splitting along $J^{\star}$. This follows from the criterion of Bochi and Gourmelon [BG, Theorem A] (see also Yoccoz [Y]). Indeed, since $f$ has constant Jacobian, for $x \in J^{\star}$ the singular values of $D f_{x}^{n}$ are $\sigma_{n}^{+}$and $\sigma_{n}^{-}=J^{n} / \sigma_{n}^{+}$, where $J=|\operatorname{Jac}(f)|<1$, and $\sigma_{n}^{+} \geq\left(C_{\eta}\right)^{-2}$. Therefore

$$
\frac{\sigma_{n}^{+}}{\sigma_{n}^{-}}=\frac{\left(\sigma_{n}^{+}\right)^{2}}{J^{n}} \geq \frac{1}{C_{\eta}^{4} J^{n}}
$$

so [BG] applies and we get a dominated splitting on $J^{\star}$. Applying Proposition 2.15 concludes the proof.

Remark 2.19. If $J^{-}$is globally laminated (outside a finite set of periodic points, say) one might expect that the additional assumption that $\mathcal{W}^{u}(x) \simeq \mathbb{C}$ for every $x$ in Theorem 2.18 would follow from the density of unstable manifolds of saddle points. Unfortunately, there are examples of minimal Riemann surface laminations containing both parabolic and hyperbolic leaves (see [Gh, Theorem 6.6])

\subsection{Concluding remarks}

2.4.1. Uniform $s$-regularity on $J^{\star}$ does not imply hyperbolicity. Indeed, there are examples of Hénon mappings with parabolic points and a dominated splitting on $J^{\star}$ (see [RT, LP]). It would be interesting to know whether uniform $s$-regularity on $J^{\star}$ implies the existence of a dominated splitting.

2.4.2. The only property of $J^{\star}$ that was used in the various hyperbolicity criteria in this section is that $J^{\star}$ is a closed invariant set in which saddle periodic points are dense. So in all these results we could work with an arbitrary closed invariant set $\Lambda$, in which saddle points are dense. The notion of uniform $u$-regularity has to be replaced by uniform $u$-regularity along $\Lambda$, meaning that the uniform size of unstable manifolds holds only for sequences of saddle points in $\Lambda$, and likewise for $s$-regularity. Then there are statements analogous to Theorems $2.8,2.12,2.13$ and 2.18, in which uniform regularity is replaced by uniform regularity along $\Lambda$, and the conclusion is that $\Lambda$ is a hyperbolic set.

\section{A topological criterion for hyperbolicity}

In this section we work in the setting of Conjecture 1.4 , We assume that $f_{0}$ and $f_{1}$ are two complex Hénon maps such that $f_{0}$ is hyperbolic, and that there exist respective neighborhoods $N_{0}$ and $N_{1}$ of $J_{0}=J_{0}^{\star}$ and $J_{1}^{\star}$ and a conjugating homeomorphism $\phi$ : $N_{0} \rightarrow N_{1}$. Our purpose is to show that $f_{1}$ is hyperbolic on $J_{1}^{\star}$.

\subsection{Periodic points and their (un)stable manifolds}

Proposition 3.1. Let $f_{0}$ and $f_{1}$ be as in Conjecture 1.4. Then $\phi\left(J_{0}\right)=J_{1}^{\star}$. If $f_{1}$ is dissipative then all periodic points of $f_{1}$ on $J_{1}^{\star}$ are saddles. If $f_{1}$ is conservative the same holds provided $\phi$ is Hölder continuous. 
Proof. The first assertion is a direct consequence of the equidistribution of periodic orbits. Indeed, the topological conjugacy shows that $f_{0}$ and $f_{1}$ have the same entropy, hence the same dynamical degree. Since periodic orbits equidistribute toward the maximal entropy measure, we get that $\phi_{\star} \mu_{0}=\mu_{1}$. Since $\operatorname{Supp}\left(\mu_{1}\right)=J_{1}^{\star}$, we infer that $\phi\left(J_{0}\right)=J_{1}^{\star}$. (On the other hand, it is unclear at this stage whether $J_{1}^{\star}=J_{1}$.)

Any periodic point on $J_{1}^{\star}$ admits a neighborhood in which it is topologically conjugate to a saddle. Let $p \in J_{1}^{\star}$ be some periodic point which we may suppose fixed. Assume that $f_{1}$ is dissipative. Then if $p$ is not a saddle it is semi-attracting, that is, its eigenvalues are $\lambda$ and $\sigma$ with $|\sigma|<1$ and $|\lambda|=1$. Then there are three possibilities: either $\lambda$ is a root of unity and by definition $p$ is semi-parabolic, or $\lambda$ is not a root of unity and $p$ is semi-Siegel if it is linearizable, and semi-Cremer otherwise. The semi-Siegel case is excluded because a semi-Siegel point belongs to the Fatou set (see [BS2]). The semi-parabolic case is excluded because a semi-parabolic point admits an open basin of attraction (see [U]) so it cannot be locally topologically conjugate to a saddle point. For the semi-Cremer case we use the hedgehog theory of [FLRT, LRT]. Indeed, in this case there exists in some neighborhood of $p$ a non-trivial totally invariant set $\mathcal{H}$ made of points which do not converge to $p$ under backward or forward iteration; more precisely, there is a subsequence $q_{n}$ such that $f^{q_{n}} \rightarrow$ id on $\mathcal{H}$ (see [FLRT, Theorem A] and [LRT, Theorem B]). This is not compatible with the local conjugacy to a saddle fixed point, therefore we conclude that $p$ is a saddle.

If $f$ is conservative and $p$ is not a saddle, then it is neutral. Since $\phi$ is Hölder, then points in $\phi\left(W_{\mathrm{loc}}^{s}\left(\phi^{-1}(p)\right)\right)$ converge to the origin exponentially fast. On the other hand, for every $\varepsilon>0$ there exists a norm on $\mathbb{C}^{2}$ for which

$$
(1-\varepsilon)\|v\| \leq\left\|d f_{p}(v)\right\| \leq(1+\varepsilon)\|v\| .
$$

Indeed, if $d f_{p}$ is diagonalizable this is clear since the eigenvalues have modulus 1 , and otherwise we can make $d f_{p}$ triangular with the off-diagonal term as small as we wish, and take an adapted norm. Thus if $x$ is close to $p$ and $f_{1}^{n}(x) \rightarrow p$ we infer that $\| f^{n}(x)-$ $f^{n}(p)\left\|\geq(1-2 \varepsilon)^{n}\right\| x-p \|$, which is contradictory if $\varepsilon$ is small enough. Hence again we conclude that all periodic points on $J_{1}^{\star}$ are saddles.

For a saddle point $p$, we now denote by $W_{r}^{s}(p)$ the component of $W^{s}(p) \cap B(p, r)$ containing $p$. We fix $r_{0}$ such that for every $x \in J_{0}, W_{r_{0}}^{s}(x)$ (respectively, $W_{r_{0}}^{u}(x)$ ) is a properly embedded holomorphic disk with the property that there exist uniform $C>0$ and $0<\lambda<1$ such that for every $x^{\prime} \in W_{r_{0}}^{s}(x)$ (respectively, $\left.W_{r_{0}}^{u}(x)\right), \operatorname{dist}\left(f^{n}(x), f^{n}\left(x^{\prime}\right)\right) \leq$ $C \lambda^{|n|}$ when $n \rightarrow \infty$ (respectively, $n \rightarrow-\infty$ ). We also assume for further reference that $f$ has product structure in the $2 r_{0}$-neighborhood of $J_{0}^{\star}$.

Proposition 3.2. Let $f_{0}$ and $f_{1}$ be as in Conjecture 1.4. There exists $r_{1}>0$ such that for any saddle periodic point $p$ for $f_{1}, W_{r_{1}}^{s}(p)$ (respectively, $\left.W_{r_{1}}^{u}(p)\right)$ is a submanifold of $B\left(p, r_{1}\right)$.

Proof. Without loss of generality we treat the case of stable manifolds and assume that $p$ is fixed. If $r_{0}$ is as above there exists $r_{1}>0$ such that for every $y \in J_{1}^{\star}, B\left(y, r_{1}\right) \Subset$ 


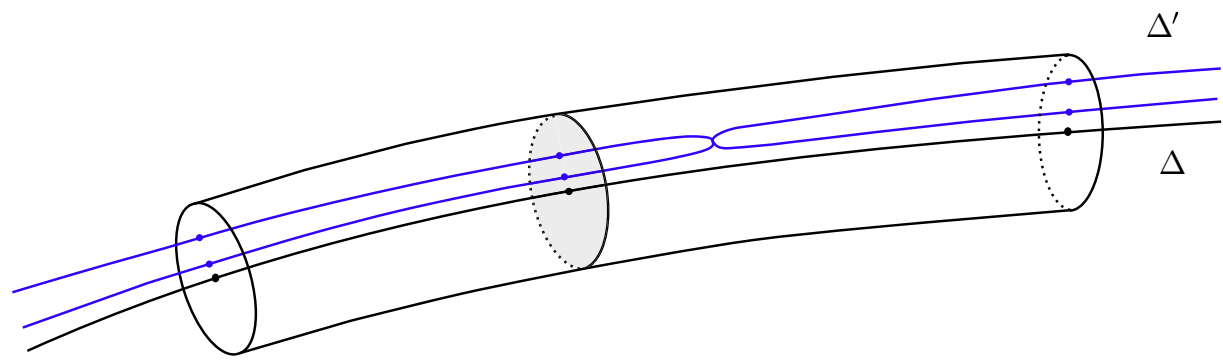

FIGURE 1. Tubular neighborhood lemma.

$\phi\left(B\left(\phi^{-1}(y), r_{0}\right)\right)$. We claim that for every saddle fixed point $p$ for $f_{1}$,

$$
W_{r_{1}}^{s}(p) \subset \phi\left(W_{r_{0}}^{s}\left(\phi^{-1}(p)\right)\right) \subset W^{s}(p)
$$

The right inclusion is obvious since belonging to $W^{s}(p)$ is characterized by the topological property that $f_{1}^{n}(y) \rightarrow p$. For the left inclusion, just observe that $\phi^{-1}\left(W_{r_{1}}^{s}(p)\right)$ is a connected subset of $W^{s}\left(\phi^{-1}(p)\right) \cap B\left(\phi^{-1}(p), r_{0}\right)$ containing $p$, hence it is contained in $W_{r_{0}}^{s}\left(\phi^{-1}(p)\right)$.

To show that it is properly embedded, we first observe that there exists $r=r(p)$ such that $W_{r}^{s}(p)$ is properly embedded in $B(p, r)$. By the invariance of domain theorem, $\phi^{-1}\left(W_{r}^{s}(p)\right)$ is a neighborhood of $\phi^{-1}(p)$ in $W^{s}\left(\phi^{-1}(p)\right)$. Thus it follows that there exists $n=n(p)$ such that $f_{0}^{n}\left(W_{r_{0}}^{s}\left(\phi^{-1}(p)\right)\right) \subset \phi^{-1}\left(W_{r}^{s}(p)\right)$. Then from (1) and topological conjugacy we get that $f_{1}^{n}\left(W_{r_{1}}^{s}(p)\right) \subset W_{r}^{s}(p)$, so $W_{r_{1}}^{s}(p) \subset f_{1}^{-n} W_{r}^{s}(p)$. From this we conclude that $W_{r_{1}}^{s}(p)$ is properly embedded in $B\left(p, r_{1}\right)$, as desired.

Remark 3.3. At this stage we know that stable manifolds are properly embedded in a ball of uniform size, but since in the last argument the quantities $n$ and $r$ are a priori not uniform in $p$, we have no uniformity for the geometry of $W_{r_{1}}^{s}(p)$. Obtaining such a uniformity will be the purpose of the forthcoming arguments.

\subsection{Tube argument.}

LEMMA 3.4. (Tubular neighborhood lemma; see Figure 1) If $\Delta$ is a submanifold in $B(0,2 r)$ of size $r$ at 0 then there exists $\eta=\eta(r)$ such that if $\Delta^{\prime}$ is a subvariety in $B(0,2 r)$ such that $d_{H}\left(\Delta, \Delta^{\prime}\right)<\eta$ in $B(0,2 r)$, then $\Delta^{\prime}$ is a branched cover over $\Delta$ in $B(0, r / 2)$. (Here $d_{H}$ denotes the Hausdorff distance.)

Proof. After a unitary change of coordinates, $\Delta$ is a graph $y=\psi(x)$ of slope at most 1 in the bidisk $D(0, r)^{2}$. Since $\psi^{\prime}(0)=0$ by the Schwarz lemma we have $\left|\psi^{\prime}(x)\right| \leq|x| / r$ so actually $|\psi(x)| \leq r / 2$. It follows that if $\eta<r / 4$ and $d_{H}\left(\Delta, \Delta^{\prime}\right)<\eta$ in $D(0, r)^{2}, \Delta^{\prime}$ is horizontal in this bidisk. Thus it is a branched cover over the first coordinate, hence over $\Delta$. 
The 'tube argument' alluded to in the title consists in applying the previous lemma to construct invariant laminations in the setting of the conjecture. Here is a sample statement.

Proposition 3.5. Let $f_{0}$ and $f_{1}$ be as in Conjecture 1.4. Then every saddle periodic point $p$ for $f_{1}$ is uniformly transverse regular.

Proof. Let $p$ be as in the statement of the proposition, and assume that $W^{s}(p)$ has size $r$ at $p$. Reducing $r$ if necessary, we may assume that $\phi^{-1}\left(W_{r}^{s}(p)\right)$ is contained in a flow box of the stable lamination of $f_{0}$. Without loss of generality we may also assume that $r<r_{1} / 4$, where $r_{1}$ is as in Proposition 3.2. We will show that there exists a neighborhood $V$ of $p$ such that if $q \in V$ is another periodic point, then $W_{r}^{s}(q)$ is a graph over $W^{s}(p)$ in $B(0, r / 2)$. This implies that $p$ is uniformly $s$-regular. Uniform $u$-regularity is proven in the same way, and the transversality property is obvious since $p$ is a saddle.

We know that for any saddle point $q \in J_{1}^{\star}$, the stable manifold $W_{r_{1}}^{s}(q)$ is properly embedded in $B\left(q, r_{1}\right)$. In addition, by (1), it is contained in $\phi\left(W_{r_{0}}^{s}\left(\phi^{-1}(q)\right)\right)$. By the uniform continuity of $\phi$ there exists a neighborhood $V$ of $p$ such that for every saddle point $q \in V, W_{r}^{s}(q)$ is $\eta$-close to $W_{r}^{s}(p)$ in $B(p, 2 r)$, where $\eta$ is as in the tubular neighborhood lemma. Thus $W_{r}^{S}(q)$ is a branched cover over $W_{r}^{s}(p)$ in $B(p, r / 2)$, and to conclude the proof it remains to show that this cover has degree 1 . By the product structure of $f_{0}$ in $\left(J_{0}^{\star}\right)_{2 r_{0}}$ we have that $W_{r_{0}}^{s}\left(\phi^{-1}(p)\right) \cap W_{r_{0}}^{u}\left(\phi^{-1}(p)\right)=\left\{\phi^{-1}(p)\right\}$, hence $W_{r_{1}}^{s}(p) \cap$ $W_{r_{1}}^{u}(p)=\{p\}$. Thus, reducing $\eta$ if necessary, to compute the degree of this branched cover it is enough to count the number of intersection points, with multiplicity, between $W_{r}^{s}(q)$ and $W_{r_{1}}^{u}(p)$. Applying the product structure again, we get that $W_{r}^{s}(q) \cap W_{r_{1}}^{u}(p)$ is a single point. Furthermore, it is well known that the order of contact between two smooth complex curves in $\mathbb{C}^{2}$ is a topological invariant. Indeed, if we consider two smooth curves $C$ and $D$ with an isolated intersection at $0 \in \mathbb{C}^{2}$ and intersect them with a small sphere $\mathbb{S}$ about 0 , then $C \cap \mathbb{S}$ winds $n$ times about $D \cap \mathbb{S}$. where $n$ is the intersection multiplicity. So we conclude that the intersection $W_{r}^{s}(q) \cap W_{r_{1}}^{u}(p)$ is transverse and we are done.

\subsection{Proof of Conjecture 1.4 in the dissipative case.}

THEOREM 3.6. Let $f_{0}$ and $f_{1}$ be two polynomial automorphisms of $\mathbb{C}^{2}$ with non-trivial dynamics, and assume that $f_{0}$ is hyperbolic and that $f_{1}$ is dissipative.

Suppose that there exist respective neighborhoods $N_{0}$ and $N_{1}$ of $J_{0}=J_{0}^{\star}$ and $J_{1}^{\star}$ and a homeomorphism $\phi: N_{0} \rightarrow N_{1}$ such that $\phi \circ f_{0}=f_{1} \circ \phi$ where these compositions make sense. Then $f_{1}$ is hyperbolic.

Proof. To prove the theorem, let $\mathcal{W}_{0}^{s}$ and $\mathcal{W}_{0}^{u}$ be respectively the stable and unstable laminations in $N_{0}$ and $\mathcal{L}_{1}^{s}$ and $\mathcal{L}_{1}^{u}$ be their respective images under $\phi$. At this stage $\mathcal{L}_{1}^{s}$ and $\mathcal{L}_{1}^{u}$ are topological laminations by topological disks in $N_{1}$.

Define $\Omega$ to be the set of points $x \in J_{1}^{\star}$ such that there exists a neighborhood $V$ of $x$ in $J_{1}^{\star}$ such that for every $y \in V, \mathcal{L}_{1}^{s}(y)$ and $\mathcal{L}_{1}^{u}(y)$ are holomorphic and of uniform size in $V$. 
Note that they must be transverse by the topological invariance of the order of contact between smooth curves. By construction, $\Omega$ is open in $J_{1}^{\star}$ and completely invariant (that is, $f(\Omega)=\Omega$ ). Proposition 3.5 shows that $\Omega$ contains all saddle points.

The main step of the proof is the following lemma.

LEMMA 3.7. Let $f_{0}$ and $f_{1}$ be as in Theorem 3.6. Then any invariant measure supported on $J_{1}^{\star}$ gives full mass to $\Omega$.

Theorem 3.6 follows easily. Indeed, if non-empty, the complement of $\Omega$ in $J_{1}^{\star}$ is a closed invariant set, hence if it is non-empty it supports an invariant measure $v$. By the lemma, $v(\Omega)=1$, hence the contradiction. Therefore we conclude that $\Omega=J_{1}^{\star}$, in particular all points in $J_{1}^{\star}$ are uniformly regular, and the result follows from Theorem 2.8.

Proof of Lemma 3.7. The method is to adapt the 'tube argument' to Pesin stable manifolds. Under the assumptions of the theorem, let $v$ be any invariant measure for $f_{1}$ supported on $J_{1}^{\star}$. Then by the Oseledets theorem for $v$-a.e. $x$ there exist Lyapunov exponents $\chi_{1}(x) \leq$ $\chi_{2}(x)$ satisfying $\chi_{1}(x)+\chi_{2}(x)=\log |\operatorname{Jac}(f)|$. In addition, since $v$ is not concentrated on a periodic orbit we have $\chi_{2}(x) \geq 0$ almost everywhere, hence $\chi_{1}(x)<0$ since $|\operatorname{Jac}(f)|<$ 1. By the Pesin stable manifold theorem, for $v$-a.e. $x$, there exists a local stable manifold $W_{\text {loc }}^{s}(x)$ which can characterized as the set of points $y$ sufficiently close to $x$ such that $\lim \sup \frac{1}{n} \log \operatorname{dist}\left(f_{1}^{n}(y), f_{1}^{n}(x)\right)<0$. Pick any point $x$ such that $W_{\text {loc }}^{s}(x)$ exists. We will show that both $\mathcal{L}_{1}^{s}$ and $\mathcal{L}_{1}^{u}$ are laminations by Riemann surfaces near $x$.

Observe first that by hyperbolicity of $f_{0}$, the local stable manifold of $\phi^{-1}(x)$ is the set of points $z$ near $\phi^{-1}(x)$ such that $\operatorname{dist}\left(f_{0}^{n}(z), f_{0}^{n}\left(\phi^{-1}(x)\right)\right) \rightarrow 0$ as $n \rightarrow+\infty$. Hence $\phi^{-1}\left(W_{\mathrm{loc}}^{s}(x)\right) \subset W_{\mathrm{loc}}^{s}\left(\phi^{-1}(x)\right)$. Since $\phi^{-1}$ is continuous and injective, by the invariance of domain theorem, $\phi^{-1}\left(W_{\mathrm{loc}}^{s}(x)\right)$ is neighborhood of $\phi^{-1}(x)$ in $W_{\mathrm{loc}}^{s}\left(\phi^{-1}(x)\right)$. Thus $W_{\mathrm{loc}}^{s}(x)$ coincides with $\mathcal{L}_{1}^{s}(x)$ in a neighborhood of $x$.

Let $r$ be so small that $W_{\text {loc }}^{s}(x)$ has size $r$ at $x$ and $W_{\text {loc }}^{s}(x)=\mathcal{L}_{1}^{s}(x)$ in $B(x, 2 r)$. Then after a unitary change of coordinates as in Lemma 3.4, $W_{\mathrm{loc}}^{s}(x)$ is a graph of the form $y=\psi(x)$ over $D(0, r)$. Denote this graph by $\Delta_{r}^{s}$. For small $\eta>0$, we define

$$
\operatorname{Tub}_{\eta}=\operatorname{Tub}_{\eta}\left(\Delta_{r}^{s}\right)=\{(x, y),|x|<r,|y-\psi(x)|<\eta\} .
$$

We say that a submanifold $M$ of $\operatorname{Tub}_{\eta}$ (which extends to some neighborhood of $\overline{\operatorname{Tub}_{\eta}}$ ) is horizontal if

$$
M \cap \partial \operatorname{Tub}_{\eta} \subset\left\{(x, y) \in \overline{\operatorname{Tub}_{\eta}},|x|=r\right\},
$$

and similarly it is vertical if $M \cap \partial \operatorname{Tub}_{\eta} \cap\{|x|=r\}=\emptyset$. As already observed, if $M$ is horizontal it is a branched covering over the first coordinate, and similarly if it is vertical the restriction of $(x, y) \mapsto y-\psi(x)$ to $M \cap \mathrm{Tub}_{\eta}$ is a branched covering over $D(0, \eta)$.

Exactly as in Proposition 3.5, if $q \in J^{\star}$ is a saddle point sufficiently close to $x$, $W_{\text {loc }}^{s}(q)$ is horizontal in $\operatorname{Tub}_{\eta}$. Now by the transversality of $\mathcal{W}_{0}^{s}$ and $\mathcal{W}_{0}^{u}$, there exists a neighborhood $N$ of $\phi^{-1}(x)$ such that for any $z \in N$, the distance between $\mathcal{W}_{0}^{u}(z)$ and $\phi^{-1}\left(\partial \Delta_{r}^{s}\right)$ is bounded from below by a uniform positive constant. By continuity of $\phi$, for 
any $y=\phi(z) \in \phi(N)$ and reducing $\eta$ if necessary we get that $\operatorname{dist}\left(\partial \Delta_{r}^{s}, \mathcal{L}_{1}^{u}(y)\right)>2 \eta$. By Proposition 3.2, if $q$ is a saddle point close to $x, W_{r_{1}}^{u}(q)=\mathcal{L}_{1}^{u}(q) \cap B\left(q, r_{1}\right)$ is a submanifold in $B\left(q, r_{1}\right)$ for a uniform $r_{1}$ (which we may assume to be large with respect to $r$ and $\eta$ ). So we conclude that it is a submanifold in a neighborhood of $\overline{\text { Tub }_{\eta}}$, which must be vertical in $\operatorname{Tub}_{\eta}$. Thus we have shown that if $q$ is a saddle periodic point sufficiently close to $x$, the local stable and unstable manifolds of $q$ are respectively horizontal and vertical in $\mathrm{Tub}_{\eta}$, with a single transverse intersection point (for transversality again we use the topological invariance of the order of contact). Hence both have covering degree 1 respectively over the horizontal and vertical directions in $\mathrm{Tub}_{\eta}$, that is, they are graphs. Then by the Schwarz lemma they have uniformly bounded geometry. So we conclude that $x$ belongs to $\Omega$, and the proof is complete.

3.4. The conservative case. In the conservative case we can only prove Conjecture 1.4 in the case of a Hölder conjugacy.

THEOREM 3.8. Let $f_{0}$ and $f_{1}$ be two polynomial automorphisms of $\mathbb{C}^{2}$ with non-trivial dynamics, and assume that $f_{0}$ is hyperbolic and $f_{1}$ is conservative.

Suppose that there exist respective neighborhoods $N_{0}$ and $N_{1}$ of $J_{0}=J_{0}^{\star}$ and $J_{1}^{\star}$ and a Hölder continuous homeomorphism $\phi: N_{0} \rightarrow N_{1}$ such that $\phi \circ f_{0}=f_{1} \circ \phi$ where these compositions make sense. Then $f_{1}$ is hyperbolic.

The proof is almost identical to that of Theorem 3.8, the only difference being that in Lemma 3.7 we need a different argument to show that any ergodic invariant measure $v$ for $f_{0}$ admits a negative Lyapunov exponent (this issue already appeared in the proof of Proposition 3.1). So Theorem 3.8 follows from the following lemma.

LEMMA 3.9. Let $f_{0}$ and $f_{1}$ be as in Theorem 3.8. Then all measures invariant under $f_{1}$ are hyperbolic.

Proof. This follows from standard Pesin-theoretic considerations. Let $v$ be an invariant measure for $f_{1}$. Without loss of generality we can assume that $v$ is ergodic so it admits two Lyapunov exponents $\chi_{1} \leq \chi_{2}$ with $\chi_{1}+\chi_{2}=0$. Assume by way of contradiction that $\chi_{1}=\chi_{2}=0$. The Oseledets-Pesin reduction theorem (see [KM, Theorem S.2.10]; note that it does not require $v$ to be hyperbolic) asserts that for every $\varepsilon>0$ there exists a measurable cocycle $C_{\varepsilon}$ with values in $\mathrm{GL}_{2}(\mathbb{C})$ such that for $v$-a.e. $x$, the matrix $A_{\varepsilon}(x):=$ $C_{\varepsilon}\left(f_{1}(x)\right)^{-1} \cdot\left(D f_{1}\right)_{x} \cdot C_{\varepsilon}(x)$ satisfies $e^{-\varepsilon} \leq\left\|A_{\varepsilon}(x)\right\| \leq e^{\varepsilon}$ and $e^{-\varepsilon} \leq\left\|\left(A_{\varepsilon}(x)\right)^{-1}\right\| \leq$ $e^{\varepsilon}$. Then the Pesin theorem on the existence of regular neighborhoods (see [KM, Theorem S.3.1]) implies that there is a measurable function $q$ such that for $v$-a.e. $x, f$ behaves likes $\left(D f_{1}\right)_{x}$ on $B(x, q(x))$ and, furthermore, $e^{-\varepsilon}<q\left(f_{1}(x)\right) / q(x)<e^{\varepsilon}$. More precisely, there is a change of coordinates $\Psi_{x}$ defined on $B(x, q(x))$ such that $\Psi_{f_{1}(x)} \circ f \circ \Psi_{x}$ is $\varepsilon$ $C^{1}$-close to its differential at $x$, which equals $A_{\varepsilon}(x)$.

Now by the Hölder conjugacy to $f_{0}$, for every $x$ there exists $y$ close to $x$ such that $\operatorname{dist}\left(f_{1}^{n}(y), f_{1}^{n}(x)\right)$ decreases like $e^{-\alpha n}$ for some $\alpha>0$. If we pick $\varepsilon$ small as compared to $\alpha$, then if $x$ satisfies the above generic requirements, we have that $f_{1}^{n}(y) \in$ 
$B\left(f^{n}(x), q\left(f^{n}(x)\right)\right.$ for every large $n$. It follows that for large $k$,

$$
\operatorname{dist}\left(f_{1}^{n+k}(y), f_{1}^{n+k}(x)\right) \geq C e^{-2 \varepsilon k} \operatorname{dist}\left(f_{1}^{n}(y), f_{1}^{n}(x)\right),
$$

which is contradictory, and the proof is complete.

\section{REFERENCES}

[BD] P. Berger and R. Dujardin. On stability and hyperbolicity for polynomial automorphisms of $\mathbb{C}^{2}$. Ann. Sci. Éc. Norm. Supér. 50(4) (2017), 449-477.

[BDV] C. Bonatti and L. Díaz and F. Vuillemin. Cubic tangencies and hyperbolic diffeomorphisms. Bull. Braz. Math. Soc. (N.S.) 29(1) (1998), 99-144.

[BG] J. Bochi and N. Gourmelon. Some characterizations of domination. Math. Z. 263(1) (2009), 221-231.

[BGS] E. Bedford, L. Guerini and J. Smillie. Hyperbolicity and quasi-hyperbolicity in polynomial diffeomorphisms of $\mathbb{C}^{2}$. Preprint, 2020, arxiv:1601.06268.

[BLS1] E. Bedford, M. Lyubich and J. Smillie. Polynomial diffeomorphisms of $\mathbb{C}^{2}$. IV. The measure of maximal entropy and laminar currents. Invent. Math. 112 (1993), 77-125.

[BLS2] E. Bedford, M. Lyubich and J. Smillie. Distribution of periodic points of polynomial diffeomorphisms of $\mathbb{C}^{2}$. Invent. Math. 114 (1993), 277-288.

[BS1] E. Bedford and J. Smillie. Polynomial diffeomorphisms of $\mathbb{C}^{2}$ : currents, equilibrium measure and hyperbolicity. Invent. Math. 103 (1991), 69-99.

[BS2] E. Bedford and J. Smillie. Polynomial diffeomorphisms of $\mathbb{C}^{2}$. II: Stable manifolds and recurrence. $J$. Amer. Math. Soc. 4 (1991), 657-679.

[BS8] E. Bedford and J. Smillie. Polynomial diffeomorphisms of $\mathbb{C}^{2}$. VIII: Quasi-expansion. Amer. J. Math. 124 (2002), 221-271.

[D1] R. Dujardin. A closing lemma for polynomial automorphisms of $\mathbb{C}^{2}$. Astérisque 415 (2020), 35-43. Quelques aspects de la théorie des systèmes dynamiques: un hommage à Jean-Christophe Yoccoz. I.

[D2] R. Dujardin. Saddle hyperbolicity implies hyperbolicity for polynomial automorphisms of $\mathbb{C}^{2}$. Math. Res. Lett. 27 (2020), 693-709

[E] H. Enrich. A heteroclinic bifurcation of Anosov diffeomorphisms. Ergod. Th. \& Dynam. Sys. 18(3) (1998), 567-608.

[F] T. Fisher. Some results in hyperbolic dynamics. PhD Thesis, Penn. State, 2006.

[FHY] A. Fathi, M. R. Herman and J.-C. Yoccoz. A proof of Pesin's stable manifold theorem. Geometric Dynamics (Rio de Janeiro, 1981) (Lecture Notes in Mathematics, 1007). Springer, Berlin, 1983, pp. 177-215.

[FLRT] T. Firsova, M. Lyubich, R. Radu and R. Tanase. Hedgehogs for neutral dissipative germs of holomorphic diffeomorphisms of $\left(\mathbb{C}^{2}, 0\right)$. Astérisque 416 (2020), 193-211. Quelques aspects de la théorie des systèmes dynamiques: un hommage à Jean-Christophe Yoccoz.II.

[FM] S. Friedland and J. Milnor. Dynamical properties of plane polynomial automorphisms. Ergod. Th. \& Dynam. Sys. 9(1) (1989), 67-99.

[Gh] É. Ghys. Laminations par surfaces de Riemann. Dynamique et géométrie complexes (Lyon, 1997) (Panoramas et Synthèses, 8). Société Mathématique de France, Paris, 1999, pp. ix, xi, 49-95.

[Go] A. Gogolev. Diffeomorphisms Hölder conjugate to Anosov diffeomorphisms. Ergod. Th. \& Dynam. Sys. 30(2) (2010), 441-456.

[GP] L. Guerini and H. Peters. Julia sets of complex Hénon maps. Internat. J. Math. 29(7) (2018), 1850047, 22.

[HOV] J. H. Hubbard and R. W. Oberste-Vorth. Hénon mappings in the complex domain. II. Projective and inductive limits of polynomials. Real and Complex Dynamical Systems (NATO Advanced Science Institutes Series C: Mathematical and Physical Sciences, 464). Kluwer Academic Publishers, Dordrecht, 1995, pp. 89-132.

[I1] Y. Ishii. Hyperbolic polynomial diffeomorphisms of $\mathbb{C}^{2}$. I. A non-planar map. Adv. Math. 218(2) (2008), 417-464.

[I2] Y. Ishii. Dynamics of polynomial diffeomorphisms of $\mathbb{C}^{2}$ : combinatorial and topological aspects. Arnold Math. J. 3 (2017), 119-173.

[IS] Y. Ishii and J. Smillie. Homotopy shadowing. Amer. J. Math. 132(4) (2010), 987-1029.

[K] A. Katok. Bernoulli diffeomorphisms on surfaces. Ann. of Math. (2) 110(3) (1979), 529-547. 
[KM] A. Katok and L. Mendoza. Dynamical systems with non-uniformly hyperbolic behavior. Appendix to Introduction to the Modern Theory of Dynamical Systems (Encyclopedia of Mathematics and its Applications, 54). Cambridge University Press, Cambridge, 1995.

[LM] M. Lyubich and Y. Minsky. Laminations in holomorphic dynamics. J. Differential Geom. 47 (1997), 17-94.

[LP] M. Lyubich and H. Peters. Structure of partially hyperbolic Hénon maps. Preprint, 2017, arxiv:1712.05823, J. Eur. Math. Soc. to appear.

[LRT] M. Lyubich, R. Radu and R. Tanase. Hedgehogs in higher dimension and their applications. Astérisque 416 (2020), 213-251. Quelques aspects de la théorie des systèmes dynamiques: un hommage à Jean-Christophe Yoccoz. II.

[MSS] R. Mañé, P. Sad and D. Sullivan. On the dynamics of rational maps. Ann. Sci. Éc. Norm. Supér. 16 (1983), 193-217.

[RT] R. Radu and R. Tanase. A structure theorem for semi-parabolic Hénon maps. Adv. Math. 350 (2019), 1000-1058.

[S] M. Sambarino. A (short) survey on dominated splittings. Mathematical Congress of the Americas (Contemporary Mathematics, 656). American Mathematical Society, Providence, RI, 2016, pp. 149-183.

[U] T. Ueda. Local structure of analytic transformations of two complex variables. I. J. Math. Kyoto Univ. 26(2) (1986), 233-261

[Y] J.-C. Yoccoz. Some questions and remarks about $S L(2, \mathbb{R})$ cocycles. Modern Dynamical Systems and Applications. Cambridge University Press, Cambridge, 2004, pp. 447-458. 